\title{
ON 4-DIMENSIONAL LORENTZIAN AFFINE HYPERSURFACES WITH AN ALMOST SYMPLECTIC FORM
}

\author{
MICHAL SZANCER AND ZUZANNA SZANCER
}

\begin{abstract}
In this paper we study 4-dimensional affine hypersurfaces with a Lorentzian second fundamental form additionally equipped with an almost symplectic structure $\omega$. We prove that the rank of the shape operator is at most one if $R^{k} \cdot \omega=0$ or $\nabla^{k} \omega=0$ for some positive integer $k$. This result is the final step in a classification of Lorentzian affine hypersurfaces with higher order parallel almost symplectic forms.
\end{abstract}

\section{INTRODUCTION}

Parallel structures are of great interest in classical Riemmanian geometry (see [7, 17, 4]) as well as in affine differential geometry ([2, 9, 10, 13, 15, 12, 14]). Higher order parallel structures are natural generalization of parallel structures and are widely studied as well $([7,8,25,26,24])$. There exist also some classification results in context of induced almost contact and almost paracontact structures $([22,23])$.

On the other hand O. Baues and V. Cortés studied affine hypersurfaces equipped with an almost complex structure (3]). They proved that every simply connected special Kähler manifold ([11) can be realized in a canonical way as an improper affine hypersphere. In 2006 V. Cortés together with M.-A. Lawn and L. Schäfer ([5]) proved a similar result for special para-Kähler manifolds ([6]). Such hyperspheres were called by the authors special affine hyperspheres. In both cases an important role was played by the Kählerian (resp. para-Kählerian) symplectic form $\omega$. Later special affine hyperspheres were generalized by the first author in [21]. These results show that there are interesting relations between symplectic (in particular Kähler and para-Kähler) geometry and affine differential geometry.

Motivated by the above results as well as M. Kon results ([16]) the first author studied affine hypersurfaces $f: M \rightarrow \mathbb{R}^{2 n+1}$ with a transversal vector field $\xi$ additionally equipped with an almost symplectic structure $\omega$. In 19 the following result was obtained:

Theorem 1.1 (19]). Let $f: M \rightarrow \mathbb{R}^{2 n+1}$ be a non-degenerate affine hypersurface with a transversal vector field $\xi$ and an almost symplectic form $\omega$. Equality $R(X, Y) \omega=0$ for every $X, Y \in \mathcal{X}(M)$ holds if and only if $\operatorname{dim} M=2$ and $\xi$ is locally equiaffine or $\operatorname{dim} M \geq 4$ and $\nabla$ is flat.

In the case when the second fundamental form is positive definite and the transversal vector field $\xi$ is locally equiaffine the above theorem generalizes to an arbitrary power of $R$. Namely, we have

1991 Mathematics Subject Classification. Primary 53A15, Secondary 53D15.

Key words and phrases. affine hypersurface, almost symplectic structure, symplectic form, Lorentzian metric. 
Theorem 1.2 (19]). Let $f: M \rightarrow \mathbb{R}^{2 n+1}$ be a non-degenerate affine hypersurface ( $\operatorname{dim} M \geq 4)$ with a locally equiaffine transversal vector field $\xi$ and an almost symplectic form $\omega$. Additionally assume that the second fundamental form is positive definite on $M$. If $R^{l} \omega=0$ for some positive integer $l$ then $\nabla$ is flat.

As a consequence of the above theorem we obtain

Theorem 1.3 (19]). Let $f: M \rightarrow \mathbb{R}^{2 n+1}$ be a non-degenerate affine hypersurface ( $\operatorname{dim} M \geq 4)$ with a locally equiaffine transversal vector field $\xi$ and an almost symplectic form $\omega$. Additionally assume that the second fundamental form is positive definite on $M$. If $\nabla^{k} \omega=0$ for some positive integer $k$ then $\nabla$ is flat.

Later in 20, it was shown that although the above theorems are not true in general when the second fundamental form is Lorentzian, we still have strong constrains on the shape operator if only $\operatorname{dim} M \geq 6$. Namely we have the following theorems:

Theorem $1.4([20])$. Let $f: M \rightarrow \mathbb{R}^{2 n+1}(\operatorname{dim} M \geq 6$ ) be a non-degenerate affine hypersurface with a locally equiaffine transversal vector field $\xi$ and an almost symplectic form $\omega$. If $R^{k} \omega=0$ for some $k \geq 1$ and the second fundamental form is Lorentzian on $M$ (that is has signature $(2 n-1,1)$ ) then the shape operator $S$ has the rank $\leq 1$.

Theorem $1.5([20])$. Let $f: M \rightarrow \mathbb{R}^{2 n+1}$ ( $\operatorname{dim} M \geq 6$ ) be a non-degenerate affine hypersurface with a locally equiaffine transversal vector field $\xi$ and an almost symplectic form $\omega$. If $\nabla^{k} \omega=0$ for some $k \geq 1$ and the second fundamental form is Lorentzian on $M$ (that is has signature $(2 n-1,1)$ ) then the shape operator $S$ has the rank $\leq 1$.

The main purpose of this paper is to prove that Theorem 1.4 and Theorem 1.5 hold also for 4-dimensional affine hypersurfaces. Although some results obtained in 20] stay true in 4-dimensional case, the key step of proof cannot be easily repeated. Simply there is not enough "room" in 4-dimensional space and results from [20] do not provide enough information about structure of eigen values of the shape operator. For this reason in this paper we need to develop a bit different methods. In particular, we consider two separate cases and find several new properties of $R^{k} \omega$ tensor.

In Section 2 we briefly recall the basic formulas of affine differential geometry. We also recall some basic definitions from symplectic geometry that will be used later in this paper.

The Section 3 contains the main results of this paper. We show that if there exists an almost symplectic structure $\omega$ satisfying condition $R^{k} \cdot \omega=0$ or $\nabla^{k} \omega=0$ for some positive integer $k$ then the shape operator must have a very special form. More precisely, we obtain that the rank of the shape operator $S$ must be $\leq 1$ if only the transversal vector field is locally equiaffine.

\section{Preliminaries}

We briefly recall the basic formulas of affine differential geometry. For more details, we refer to [18. Let $f: M \rightarrow \mathbb{R}^{n+1}$ be an orientable connected differentiable $n$-dimensional hypersurface immersed in the affine space $\mathbb{R}^{n+1}$ equipped with its usual flat connection D. Then for any transversal vector field $\xi$ we have

$$
\mathrm{D}_{X} f_{*} Y=f_{*}\left(\nabla_{X} Y\right)+h(X, Y) \xi
$$


and

$$
\mathrm{D}_{X} \xi=-f_{*}(S X)+\tau(X) \xi
$$

where $X, Y$ are vector fields tangent to $M$. It is known that $\nabla$ is a torsion-free connection, $h$ is a symmetric bilinear form on $M$, called the second fundamental form, $S$ is a tensor of type $(1,1)$, called the shape operator, and $\tau$ is a 1-form, called the transversal connection form. The vector field $\xi$ is called equiaffine if $\tau=0$. When $d \tau=0$ the vector field $\xi$ is called locally equiaffine.

When $h$ is non-degenerate then $h$ defines a pseudo-Riemannian metric on $M$. In this case we say that the hypersurface or the hypersurface immersion is nondegenerate. In this paper we always assume that $f$ is non-degenerate. We have the following

Theorem 2.1 ([18, Fundamental equations). For an arbitrary transversal vector field $\xi$ the induced connection $\nabla$, the second fundamental form $h$, the shape operator $S$, and the 1-form $\tau$ satisfy the following equations:

$$
\begin{aligned}
& R(X, Y) Z=h(Y, Z) S X-h(X, Z) S Y, \\
& \left(\nabla_{X} h\right)(Y, Z)+\tau(X) h(Y, Z)=\left(\nabla_{Y} h\right)(X, Z)+\tau(Y) h(X, Z), \\
& \left(\nabla_{X} S\right)(Y)-\tau(X) S Y=\left(\nabla_{Y} S\right)(X)-\tau(Y) S X, \\
& h(X, S Y)-h(S X, Y)=2 d \tau(X, Y) .
\end{aligned}
$$

The equations (2.3), (2.4), (2.5), and (2.6) are called the equations of Gauss, Codazzi for $h$, Codazzi for $S$ and Ricci, respectively.

Let $\omega$ be a non-degenerate 2 -form on manifold $M$. The form $\omega$ we call an almost symplectic structure. It is easy to see that if a manifold $M$ admits some almost symplectic structure then $M$ is orientable manifold of even dimension. Structure $\omega$ is called a symplectic structure, if it is almost symplectic and additionally satisfies $d \omega=0$. Pair $(M, \omega)$ we call (almost) symplectic manifold, if $\omega$ is (almost) symplectic structure on $M$.

Recall ([1]) that affine connection $\nabla$ on an almost symplectic manifold $(M, \omega)$ we call an almost symplectic connection if $\nabla \omega=0$. An affine connection $\nabla$ on an almost symplectic manifold $(M, \omega)$ we call a symplectic connection if it is almost symplectic and torsion-free.

For a tensor field $T$ of type $(0, p)$ its covariant derivation $\nabla T$ is a tensor field of type $(0, p+1)$ given by the formula:

$$
\begin{array}{r}
(\nabla T)\left(X_{1}, X_{2}, \ldots, X_{p+1}\right):=X_{1}\left(T\left(X_{2}, \ldots, X_{p+1}\right)\right) \\
-\sum_{i=2}^{p+1} T\left(X_{2}, \ldots, \nabla_{X_{1}} X_{i}, \ldots, X_{p+1}\right) .
\end{array}
$$

Higher order covariant derivatives of $T$ can be defined by recursion:

$$
\left(\nabla^{k+1} T\right)=\nabla\left(\nabla^{k} T\right) \text {. }
$$

To simplify computation it is often convenient to define $\nabla^{0} T:=T$.

If $R$ is a curvature tensor for an affine connection $\nabla$, one can define a new tensor $R \cdot T$ of type $(0, p+2)$ by the formula

$$
(R \cdot T)\left(X_{1}, X_{2}, \ldots, X_{p+2}\right):=-\sum_{i=3}^{p+2} T\left(X_{3}, \ldots, R\left(X_{1}, X_{2}\right) X_{i}, \ldots, X_{p+2}\right) .
$$


Analogously to the previous case, we may define a tensor $R^{k} \cdot T$ of type $(0,2 k+p)$ using the following recursive formula:

$$
R^{k} \cdot T=R \cdot\left(R^{k-1} \cdot T\right)
$$

and additionally $R^{0} \cdot T:=T$.

\section{Hypersurfaces With "Higher order" PARAllel SyMPleCtiC STRUCTURE}

In this section we study properties of 4-dimensional affine hypersurfaces $f: M \rightarrow$ $\mathbb{R}^{5}$ with a Lorentzian second fundamental form. We assume that our hypersurfaces are equipped with an almost symplectic structure $\omega$ satisfying condition $R^{k} \omega=0$ for some positive integer $k$. In particular we obtain constrains on hypersurfaces with the property $\nabla^{k} \omega=0$.

First we recall the following lemma from [19].

Lemma 3.1 (19]). Let $T$ be a tensor of type $(0, p)$ and let $\nabla$ be an affine torsionfree connection. Then for every $k \geq 1$ and for any $2 k+p$ vector fields $X_{ \pm 1}^{1}, \ldots, X_{ \pm 1}^{k}$, $Y_{1}, \ldots, Y_{p}$ the following identity holds:

$$
\begin{aligned}
& \left(R^{k} \cdot T\right)\left(X_{1}^{1}, X_{-1}^{1}, \ldots, X_{1}^{k}, X_{-1}^{k}, Y_{1}, \ldots, Y_{p}\right) \\
& \quad=\sum_{a \in \mathcal{J}} \operatorname{sgn} a\left(\nabla^{2 k} T\right)\left(X_{a(1)}^{1}, X_{-a(1)}^{1}, \ldots, X_{a(k)}^{k}, X_{-a(k)}^{k}, Y_{1}, \ldots, Y_{p}\right),
\end{aligned}
$$

where $\mathcal{J}=\left\{a: I_{k} \rightarrow\{-1,1\}\right\}$ and $\operatorname{sgn} a:=a(1) \cdot \ldots \cdot a(k)$.

In order to simplify the notation, we will be often omitting "." in $R^{k} \cdot T$ when no confusion arises. Thus we will be writing often $R^{k} T$ instead of $R^{k} \cdot T$.

In all the below lemmas we assume that $f: M \rightarrow \mathbb{R}^{5}$ is a non-degenerate affine hypersurface with a locally equiaffine transversal vector field $\xi$ and an almost symplectic form $\omega$. About objects $\nabla, h, S$ and $\tau$ we assume that they are induced by $\xi$.

First note that combining Lemma 3.6 and Lemma 3.11 from [20] and adapting it to 4-dimensional case we have the following:

Lemma $3.2\left([20)\right.$. Let $f: M \rightarrow \mathbb{R}^{5}$ be a non-degenerate Lorentzian affine hypersurface with a locally equiaffine transversal vector field $\xi$ and an almost symplectic form $\omega$. If $R^{k} \omega=0$ for some $k \geq 1$ then for every point $x \in M$ there exists a basis $e_{1}, \ldots, e_{4}$ of $T_{x} M$ such that the shape operator $S$ and the second fundamental form $h$ can be expressed in this basis either in the form

$$
S=\left[\begin{array}{cccc}
\lambda_{1} & 0 & 0 & 0 \\
0 & \lambda_{2} & 0 & 0 \\
0 & 0 & \lambda_{3} & 0 \\
0 & 0 & 0 & \lambda_{4}
\end{array}\right] h=\left[\begin{array}{cccc}
1 & 0 & 0 & 0 \\
0 & 1 & 0 & 0 \\
0 & 0 & 1 & 0 \\
0 & 0 & 0 & -1
\end{array}\right],
$$

where $\lambda_{1}, \ldots, \lambda_{4} \in \mathbb{R}$, or in the form

$$
S=\left[\begin{array}{cccc}
\lambda_{1} & 0 & 0 & 0 \\
0 & \lambda_{2} & 0 & 0 \\
0 & 0 & \alpha & \gamma \\
0 & 0 & -\gamma & \beta
\end{array}\right] h=\left[\begin{array}{cccc}
1 & 0 & 0 & 0 \\
0 & 1 & 0 & 0 \\
0 & 0 & 1 & 0 \\
0 & 0 & 0 & -1
\end{array}\right],
$$

where $\lambda_{1}, \lambda_{2}, \alpha, \beta, \gamma \in \mathbb{R}, \gamma \neq 0$.

Let us recall yet another lemma from [20] (again adapted to 4-dimensional case). 
Lemma 3.3 (20]). If $S$ and $h$ are of the form (3.3) then for every $k \geq 1$ we have

$$
\begin{array}{r}
R^{2 k} \omega(\underbrace{e_{3}, e_{4}, \ldots, e_{3}, e_{4}}_{4 k}, e_{i}, e_{4}) \\
=\operatorname{det}\left[\begin{array}{cc}
\alpha & \gamma \\
-\gamma & \beta
\end{array}\right]^{k} \omega\left(e_{i}, e_{4}\right)
\end{array}
$$

if $i<3$,

$$
\begin{array}{r}
R^{2 k+1} \omega(\underbrace{e_{3}, e_{4}, \ldots, e_{3}, e_{4}}_{4 k}, e_{1}, X, e_{1}, X) \\
=4^{k} \gamma \operatorname{det}\left[\begin{array}{cc}
\alpha & \gamma \\
-\gamma & \beta
\end{array}\right]^{k} \omega\left(e_{3}, e_{4}\right)
\end{array}
$$

for $X=e_{3}$ or $X=e_{4}$,

$$
\begin{array}{r}
R^{2 k+1} \omega(\underbrace{e_{3}, e_{4}, \ldots, e_{3}, e_{4}}_{4 k}, e_{1}, X, e_{1}, Y) \\
=2 \cdot 4^{k-1}(\alpha-\beta) \operatorname{det}\left[\begin{array}{cc}
\alpha & \gamma \\
-\gamma & \beta
\end{array}\right]^{k} \omega\left(e_{3}, e_{4}\right),
\end{array}
$$

for $X=e_{3}$ and $Y=e_{4}$ or $X=e_{4}$ and $Y=e_{3}$.

Thanks to the above lemma we have the following:

Corollary 3.4. If $S$ and $h$ are of the form (3.3) and $R^{k} \omega=0$ for some $k \geq 1$ then

$$
\operatorname{det}\left[\begin{array}{cc}
\alpha & \gamma \\
-\gamma & \beta
\end{array}\right]=\alpha \beta+\gamma^{2}=0
$$

Proof. If $R^{k} \omega=0$ then $R^{2 k} \omega=0$ and $R^{2 k+1} \omega=0$. Since $\omega$ is non-degenerate we can find $i<4$ such that $\omega\left(e_{i}, e_{4}\right) \neq 0$. If $i=1$ or $i=2$ then by formula (3.4) we get $\alpha \beta+\gamma^{2}=0$. If $i=3$ then by formula (3.5) we again obtain $\alpha \beta+\gamma^{2}=0$ (since $\gamma \neq 0)$.

Now, we shall consider two separate cases: when $\beta^{2}-\gamma^{2} \neq 0$ and when $\beta^{2}-\gamma^{2}=$ 0 . In the first case, using suitable change of the basis one may show that $S$ is diagonalisable. Namely, we have

Lemma 3.5. If $S$ and $h$ are of the form (3.3) and $\beta^{2}-\gamma^{2} \neq 0$ and $R^{k} \omega=0$ for some $k \geq 1$ then there exists a basis $e_{1}^{\prime}, \ldots, e_{4}^{\prime}$ of $T_{x} M$ such that the shape operator $S$ and the second fundamental form $h$ can be expressed in this new basis in the following form:

$$
S=\left[\begin{array}{cccc}
\lambda_{1} & 0 & 0 & 0 \\
0 & \lambda_{2} & 0 & 0 \\
0 & 0 & \alpha+\beta & 0 \\
0 & 0 & 0 & 0
\end{array}\right] h=\left[\begin{array}{cccc}
1 & 0 & 0 & 0 \\
0 & 1 & 0 & 0 \\
0 & 0 & -\epsilon & 0 \\
0 & 0 & 0 & \epsilon
\end{array}\right]
$$

where $\epsilon=\operatorname{sgn}\left(\beta^{2}-\gamma^{2}\right) \in\{1,-1\}$. 
Proof. Let as define a matrix

$$
P=\left[\begin{array}{cccc}
1 & 0 & 0 & 0 \\
0 & 1 & 0 & 0 \\
0 & 0 & \frac{\gamma}{\sqrt{\left|\beta^{2}-\gamma^{2}\right|}} & \frac{\beta}{\sqrt{\left|\beta^{2}-\gamma^{2}\right|}} \\
0 & 0 & \frac{\beta}{\sqrt{\left|\beta^{2}-\gamma^{2}\right|}} & \frac{\gamma}{\sqrt{\left|\beta^{2}-\gamma^{2}\right|}}
\end{array}\right]
$$

Since $\operatorname{det} P= \pm 1$ the matrix $P$ is non-singular and we can define a new basis of $T_{x} M$ by the formula $e_{i}^{\prime}:=P e_{i}$ for $i=1, \ldots, 4$. By straightforward computations we check that $S$ and $h$ in this new basis take the form:

$$
S=\left[\begin{array}{cccc}
\lambda_{1} & 0 & 0 & 0 \\
0 & \lambda_{2} & 0 & 0 \\
0 & 0 & \frac{\beta^{3}-2 \beta \gamma^{2}-\alpha \gamma^{2}}{\beta^{2}-\gamma^{2}} & -\frac{\left(\alpha \beta+\gamma^{2}\right) \gamma}{\beta^{2}-\gamma^{2}} \\
0 & 0 & \frac{\left(\alpha \beta+\gamma^{2}\right) \gamma}{\beta^{2}-\gamma^{2}} & -\frac{\left(\alpha \beta+\gamma^{2}\right) \beta}{\beta^{2}-\gamma^{2}}
\end{array}\right] h=\left[\begin{array}{cccc}
1 & 0 & 0 & 0 \\
0 & 1 & 0 & 0 \\
0 & 0 & -\epsilon & 0 \\
0 & 0 & 0 & \epsilon
\end{array}\right]
$$

Eventually, using Corollary 3.4 we see that $S$ simplify to (3.7).

When $\beta^{2}-\gamma^{2}=0$ the situation is much more complicated. In this case we have $\alpha= \pm \gamma$ and $\beta=\mp \gamma$. Most part of this section is devoted to this case.

In order to simplify further computations, let us introduce the following notation:

$$
\begin{aligned}
& A_{k}:=R^{k} \omega(e_{1}, e_{4}, e_{3}, e_{4}, \overbrace{e_{3}, e_{4}, \ldots, e_{3}, e_{4}}^{2 k-2}) ; \\
& B_{k}:=R^{k} \omega(e_{3}, e_{4}, e_{1}, e_{4}, \overbrace{e_{3}, e_{4}, \ldots, e_{3}, e_{4}}^{2 k-2}) ; \\
& C_{k}:=R^{k} \omega(e_{1}, e_{3}, e_{3}, e_{4}, \overbrace{e_{3}, e_{4}, \ldots, e_{3}, e_{4}}^{2 k-2}) ; \\
& D_{k}:=R^{k} \omega(e_{3}, e_{4}, e_{1}, e_{3}, \overbrace{e_{3}, e_{4}, \ldots, e_{3}, e_{4}}^{2 k-2})
\end{aligned}
$$

for $k \geq 1$.

Lemma 3.6. If $S$ and $h$ are of the form (3.3) and $\alpha= \pm \gamma$ and $\beta=\mp \gamma$ then for every $k \geq 1$ we have

$$
\begin{array}{r}
B_{k+1}= \pm \gamma C_{k}-\gamma A_{k}, \\
D_{k+1}=\gamma C_{k} \mp \gamma A_{k} .
\end{array}
$$

Proof. We shall prove only (3.9). The proof of (3.10) goes in a similar way. First note, that by the Gauss equation we have

$$
\begin{array}{r}
R\left(e_{3}, e_{4}\right) e_{1}=R\left(e_{3}, e_{4}\right) e_{2}=0, \\
R\left(e_{3}, e_{4}\right) e_{3}=-S e_{4}=-\gamma e_{3} \pm \gamma e_{4}, \\
R\left(e_{3}, e_{4}\right) e_{4}=-S e_{3}=\mp \gamma e_{3}+\gamma e_{4} .
\end{array}
$$


Now we compute

$$
\begin{aligned}
B_{k+1}= & R^{k+1} \omega(e_{3}, e_{4}, e_{1}, e_{4}, \overbrace{e_{3}, e_{4}, \ldots, e_{3}, e_{4}}^{2 k}) \\
= & R\left(e_{3}, e_{4}\right) \cdot R^{k} \omega(e_{1}, e_{4}, \overbrace{e_{3}, e_{4}, \ldots, e_{3}, e_{4}}^{2 k}) \\
= & -R^{k} \omega(R\left(e_{3}, e_{4}\right) e_{1}, e_{4}, \overbrace{e_{3}, e_{4}, \ldots, e_{3}, e_{4}}^{2 k}) \\
& -R^{k} \omega(e_{1}, R\left(e_{3}, e_{4}\right) e_{4}, \overbrace{e_{3}, e_{4}, \ldots, e_{3}, e_{4}}^{2 k}) \\
& -R^{k} \omega(e_{1}, e_{4}, \overbrace{R\left(e_{3}, e_{4}\right) e_{3}, e_{4}, \ldots, e_{3}, e_{4}}^{2 k}) \\
& \ldots \\
& -R^{k} \omega(e_{1}, e_{4}, \overbrace{e_{3}, e_{4}, \ldots, e_{3}, R\left(e_{3}, e_{4}\right) e_{4}}^{2 k}) .
\end{aligned}
$$

Using (3.11) - 3.13) we obtain

$$
\begin{aligned}
B_{k+1}= & 0-R^{k} \omega(e_{1}, \mp \gamma e_{3}+\gamma e_{4}, \overbrace{e_{3}, e_{4}, \ldots, e_{3}, e_{4}}^{2 k}) \\
& -R^{k} \omega(e_{1}, e_{4}, \overbrace{-\gamma e_{3} \pm \gamma e_{4}, e_{4}, \ldots, e_{3}, e_{4}}^{2 k}) \\
& \ldots \\
& -R^{k} \omega(e_{1}, e_{4}, \overbrace{e_{3}, e_{4}, \ldots, e_{3}, \mp \gamma e_{3}+\gamma e_{4}}^{2 k}) \\
= & \pm \gamma C_{k}-\gamma A_{k} \\
& +\left(\gamma A_{k}-\gamma A_{k}\right)+\cdots+\left(\gamma A_{k}-\gamma A_{k}\right) \\
= & \pm \gamma C_{k}-\gamma A_{k} .
\end{aligned}
$$

Now, let us define a family of 2 -forms on $T_{x} M$ as follows:

$$
E_{k}^{i}(X, Y):=R^{k} \omega(\overbrace{e_{3}, e_{4}}^{1}, \overbrace{e_{3}, e_{4}}^{2}, \ldots, e_{3}, e_{4}, \overbrace{X, Y}^{i}, e_{3}, e_{4}, \ldots, e_{3}, e_{4})
$$

for $k \geq 1, i \in\{1, \ldots, k+1\}$. We have the following lemma:

Lemma 3.7. If $S$ and $h$ are of the form (3.3) and $\alpha= \pm \gamma$ and $\beta=\mp \gamma$ then for every $k \geq 2, i \in\{3, \ldots, k+1\}$ and $X, Y \in\left\{e_{1}, e_{3}, e_{4}\right\}$ we have $E_{k}^{i}(X, Y)=0$.

Proof. For $k=2$ and $i=3$ by straightforward computation we check that $E_{2}^{3}(X, Y)=$ 0 . Assume now that $E_{k}^{i}(X, Y)=0$ for some $k \geq 2$ and for every $i \in\{3, \ldots, k+1\}$. 
Let us fix $i \in\{3, \ldots, k+2\}$. Then we have

$$
\begin{aligned}
& E_{k+1}^{i}(X, Y)=R^{k+1} \omega(e_{3}, e_{4}, e_{3}, e_{4}, \ldots, e_{3}, e_{4}, \overbrace{X, Y}^{i}, e_{3}, e_{4}, \ldots, e_{3}, e_{4}) \\
& =-R^{k} \omega(R\left(e_{3}, e_{4}\right) e_{3}, e_{4}, \ldots, e_{3}, e_{4}, \overbrace{X, Y}^{i-1}, e_{3}, e_{4}, \ldots, e_{3}, e_{4}) \\
& -R^{k} \omega(e_{3}, R\left(e_{3}, e_{4}\right) e_{4}, \ldots, e_{3}, e_{4}, \overbrace{X, Y}^{i-1}, e_{3}, e_{4}, \ldots, e_{3}, e_{4}) \\
& \text {... } \\
& -R^{k} \omega(e_{3}, e_{4}, \ldots, e_{3}, e_{4}, \overbrace{R\left(e_{3}, e_{4}\right) X, Y}^{i-1}, e_{3}, e_{4}, \ldots, e_{3}, e_{4}) \\
& -R^{k} \omega(e_{3}, e_{4}, \ldots, e_{3}, e_{4}, \overbrace{X, R\left(e_{3}, e_{4}\right) Y}^{i-1}, e_{3}, e_{4}, \ldots, e_{3}, e_{4}) \\
& \text {... } \\
& -R^{k} \omega(e_{3}, e_{4}, \ldots, e_{3}, e_{4}, \overbrace{X, Y}^{i-1}, e_{3}, e_{4}, \ldots, R\left(e_{3}, e_{4}\right) e_{3}, e_{4}) \\
& -R^{k} \omega(e_{3}, e_{4}, \ldots, e_{3}, e_{4}, \overbrace{X, Y}^{i-1}, e_{3}, e_{4}, \ldots, e_{3}, R\left(e_{3}, e_{4}\right) e_{4}) \\
& =-R^{k} \omega(e_{3}, e_{4}, \ldots, e_{3}, e_{4}, \overbrace{R\left(e_{3}, e_{4}\right) X, Y}^{i-1}, e_{3}, e_{4}, \ldots, e_{3}, e_{4}) \\
& -R^{k} \omega(e_{3}, e_{4}, \ldots, e_{3}, e_{4}, \overbrace{X, R\left(e_{3}, e_{4}\right) Y}^{i-1}, e_{3}, e_{4}, \ldots, e_{3}, e_{4})
\end{aligned}
$$

where the last equality follows from (3.12) $-(3.13)$. The above formula can be rewritten as follows:

$$
E_{k+1}^{i}(X, Y)=-E_{k}^{i-1}\left(R\left(e_{3}, e_{4}\right) X, Y\right)-E_{k}^{i-1}\left(X, R\left(e_{3}, e_{4}\right) Y\right) .
$$

Let $i>3$. Taking into account that $X, Y \in\left\{e_{1}, e_{3}, e_{4}\right\}$ and using (3.11)-3.13) we obtain that $E_{k+1}^{i}(X, Y)$ can be expressed as a linear combination of $E_{k}^{i-1}(Z, W)$, where $Z, W \in\left\{e_{1}, e_{3}, e_{4}\right\}$. Since $i>3$ we have $i-1 \geq 3$ and by assumption $E_{k}^{i-1}(Z, W)=0$. Now it follows that $E_{k+1}^{i}(X, Y)=0$.

Assume now that $i=3$, then we have

$$
\begin{aligned}
E_{k+1}^{3}(X, Y)= & -R^{k} \omega\left(e_{3}, e_{4}, R\left(e_{3}, e_{4}\right) X, Y, e_{3}, e_{4}, \ldots, e_{3}, e_{4}\right) \\
& -R^{k} \omega\left(e_{3}, e_{4}, X, R\left(e_{3}, e_{4}\right) Y, e_{3}, e_{4}, \ldots, e_{3}, e_{4}\right) .
\end{aligned}
$$

For any pair $(X, Y)$ there exists $i, j \in\{1,3,4\}$ such that $X=e_{i}$ and $Y=e_{j}$ Since $E_{k+1}^{3}(X, Y)$ is antisymmetric relative to $X, Y$ it is enough to show that $E_{k+1}^{3}\left(e_{i}, e_{j}\right)=0$ for $i, j \in\{1,3,4\}, i<j$. We have the following possibilities:

(i) $(X, Y)=\left(e_{1}, e_{3}\right)$. In this case we have

$$
\begin{aligned}
E_{k+1}^{3}(X, Y) & =-R^{k} \omega\left(e_{3}, e_{4}, e_{1}, R\left(e_{3}, e_{4}\right) e_{3}, e_{3}, e_{4}, \ldots, e_{3}, e_{4}\right) \\
& =-R^{k} \omega\left(e_{3}, e_{4}, e_{1},-\gamma e_{3} \pm \gamma e_{4}, e_{3}, e_{4}, \ldots, e_{3}, e_{4}\right) \\
& =\gamma D_{k} \mp \gamma B_{k}=0,
\end{aligned}
$$


where the last equality follows from Lemma 3.6.

(ii) $(X, Y)=\left(e_{1}, e_{4}\right)$ In this case we have

$$
\begin{aligned}
E_{k+1}^{3}(X, Y) & =-R^{k} \omega\left(e_{3}, e_{4}, e_{1}, R\left(e_{3}, e_{4}\right) e_{4}, e_{3}, e_{4}, \ldots, e_{3}, e_{4}\right) \\
& =-R^{k} \omega\left(e_{3}, e_{4}, e_{1}, \mp \gamma e_{3}+\gamma e_{4}, e_{3}, e_{4}, \ldots, e_{3}, e_{4}\right) \\
& = \pm \gamma D_{k}-\gamma B_{k}=0
\end{aligned}
$$

where the last equality is also consequence of Lemma 3.6 .

(iii) $(X, Y)=\left(e_{3}, e_{4}\right)$ In this case $E_{k+1}^{3}(X, Y)=0$ thanks to (3.12) and (3.13).

Summarising we have shown that $E_{k+1}^{i}(X, Y)=0$ for all $i \in\{3, \ldots, k+2\}$. Now by induction principle $E_{k}^{i}(X, Y)=0$ for all $k \geq 2 i \in\{3, \ldots, k+1\}$ and $X, Y \in\left\{e_{1}, e_{3}, e_{4}\right\}$.

As a consequence of Lemma 3.7 one may prove the following

Lemma 3.8. If $S$ and $h$ are of the form (3.3) and $\alpha= \pm \gamma$ and $\beta=\mp \gamma$ then for every $k \geq 1, i \in\{1, \ldots, k+1\}$ and $X, Y \in\left\{e_{3}, e_{4}\right\}$ we have $E_{k}^{i}(S X, Y)=0$.

Proof. For $X \in\left\{e_{3}, e_{4}\right\}$ we have that $S X$ is a linear combination of $e_{3}$ and $e_{4}$. Since $E_{k}^{i}$ is antisymmetric 2 -form we conclude that there exists a constant $c_{0} \in \mathbb{R}$ such that

$$
E_{k}^{i}(S X, Y)=c_{0} \cdot E_{k}^{i}\left(e_{3}, e_{4}\right)
$$

Now, if $k \geq 2$ the thesis follows from Lemma 3.7. If $k=1$ we check by direct computation that $E_{1}^{i}\left(e_{3}, e_{4}\right)=0$ for $i=1,2$.

Now we are at the position to prove the following lemma:

Lemma 3.9. If $S$ and $h$ are of the form (3.3) and $\alpha= \pm \gamma$ and $\beta=\mp \gamma$ then for every $k \geq 1$ we have

$$
\begin{aligned}
& A_{k+1}=-\lambda_{1}\left(C_{k}+D_{k}\right), \\
& C_{k+1}=-\lambda_{1}\left(A_{k}+B_{k}\right) .
\end{aligned}
$$

Proof. We compute

$$
\begin{aligned}
A_{k+1}= & R^{k+1} \omega\left(e_{1}, e_{4}, e_{3}, e_{4}, \ldots, e_{3}, e_{4}\right) \\
= & R\left(e_{1}, e_{4}\right) \cdot R^{k} \omega\left(e_{3}, e_{4}, \ldots, e_{3}, e_{4}\right) \\
= & -R^{k} \omega\left(R\left(e_{1}, e_{4}\right) e_{3}, e_{4}, \ldots, e_{3}, e_{4}\right) \\
& -R^{k} \omega\left(e_{3}, R\left(e_{1}, e_{4}\right) e_{4}, \ldots, e_{3}, e_{4}\right) \\
& \ldots \\
& -R^{k} \omega\left(e_{3}, e_{4}, \ldots, e_{3}, R\left(e_{1}, e_{4}\right) e_{4}\right) .
\end{aligned}
$$


Since $R\left(e_{1}, e_{4}\right) e_{3}=0$ and $R\left(e_{1}, e_{4}\right) e_{4}=-\lambda_{1}$ the above formula can be simplified as follows:

$$
\begin{aligned}
A_{k+1}= & \lambda_{1} R^{k} \omega\left(e_{3}, e_{1}, e_{3}, e_{4}, \ldots, e_{3}, e_{4}\right) \\
& +\lambda_{1} R^{k} \omega\left(e_{3}, e_{4}, e_{3}, e_{1}, \ldots, e_{3}, e_{4}\right) \\
& \ldots \\
& +\lambda_{1} R^{k} \omega\left(e_{3}, e_{4}, e_{3}, e_{4}, \ldots, e_{3}, e_{1}\right) \\
& =\lambda_{1} \cdot \sum_{i=1}^{k+1} E_{k}^{i}\left(e_{3}, e_{1}\right) .
\end{aligned}
$$

If $k=1$ we have

$$
A_{2}=\lambda_{1}\left(E_{1}^{1}\left(e_{3}, e_{1}\right)+E_{1}^{2}\left(e_{3}, e_{1}\right)\right)=-\lambda_{1}\left(C_{1}+D_{1}\right) .
$$

If $k \geq 2$, by Lemma $3.7 E_{k}^{i}\left(e_{3}, e_{1}\right)=0$ for $i=3, \ldots, k+1$. That is we obtain

$$
A_{k+1}=\lambda_{1}\left(E_{k}^{1}\left(e_{3}, e_{1}\right)+E_{k}^{2}\left(e_{3}, e_{1}\right)\right)=-\lambda_{1}\left(C_{k}+D_{k}\right) .
$$

Eventually we have shown (3.15). The proof of (3.16) is similar.

Lemma 3.10. If $S$ and $h$ are of the form (3.3) and $\alpha= \pm \gamma$ and $\beta=\mp \gamma$ then for every $k \geq 0$ we have

$$
\begin{aligned}
& A_{2 k+1}=-\lambda_{1}^{2 k+1} \omega\left(e_{1}, e_{3}\right), \\
& C_{2 k+1}=-\lambda_{1}^{2 k+1} \omega\left(e_{1}, e_{4}\right) .
\end{aligned}
$$

Proof. By straightforward computations we get

$$
\begin{aligned}
& A_{1}=R \omega\left(e_{1}, e_{4}, e_{3}, e_{4}\right)=-\lambda_{1} \omega\left(e_{1}, e_{3}\right), \\
& B_{1}= \pm \gamma\left(\omega\left(e_{1}, e_{3}\right) \mp \omega\left(e_{1}, e_{4}\right)\right), \\
& C_{1}=R \omega\left(e_{1}, e_{3}, e_{3}, e_{4}\right)=-\lambda_{1} \omega\left(e_{1}, e_{4}\right), \\
& D_{1}=\gamma\left(\omega\left(e_{1}, e_{3}\right) \mp \omega\left(e_{1}, e_{4}\right)\right) .
\end{aligned}
$$

By Lemma 3.6 we also have

$$
\begin{aligned}
B_{k+1} & = \pm \gamma\left(C_{k} \mp A_{k}\right), \\
D_{k+1} & =\gamma\left(C_{k} \mp A_{k}\right)
\end{aligned}
$$

for all $k \geq 1$. Summarising we have

$$
D_{k}= \pm B_{k}
$$

for $k \geq 1$. Now, using Lemma 3.9 we obtain

$$
\begin{aligned}
C_{k+1} \mp A_{k+1} & =-\lambda_{1}\left(A_{k}+B_{k}\right) \pm \lambda_{1}\left(C_{k}+D_{k}\right) \\
& = \pm \lambda_{1}\left(C_{k} \mp A_{k}+D_{k} \mp B_{k}\right) \\
& = \pm \lambda_{1}\left(C_{k} \mp A_{k}\right)
\end{aligned}
$$

where the last equality is a consequence of (3.19). The above implies, that $C_{k} \mp A_{k}$ is a geometric sequence, that is for $k \geq 1$ we have

$$
C_{k} \mp A_{k}=\left( \pm \lambda_{1}\right)^{k-1}\left(C_{1} \mp A_{1}\right) .
$$


In particular we obtain explicit formulas for $B_{k+1}$ and $D_{k+1}$ :

$$
\begin{aligned}
& B_{k+1}= \pm \gamma\left( \pm \lambda_{1}\right)^{k-1}\left(C_{1} \mp A_{1}\right), \\
& D_{k+1}=\gamma\left( \pm \lambda_{1}\right)^{k-1}\left(C_{1} \mp A_{1}\right) .
\end{aligned}
$$

Using (3.15)-(3.16) and (3.21) for all $k \geq 1$ we have

$$
\begin{aligned}
A_{2 k+1} & =-\lambda_{1}\left(C_{2 k}+D_{2 k}\right) \\
& =\lambda_{1}^{2}\left(A_{2 k-1}+B_{2 k-1}\right)-\lambda_{1} D_{2 k} \\
& =\lambda_{1}^{2} A_{2 k-1}+\lambda_{1}^{2} B_{2 k-1}-\lambda_{1} \gamma\left( \pm \lambda_{1}\right)^{2 k-2}\left(C_{1} \mp A_{1}\right) \\
& =\lambda_{1}^{2} A_{2 k-1}+\lambda_{1}^{2} B_{2 k-1}-\gamma \lambda_{1}^{2 k-1}\left(C_{1} \mp A_{1}\right) .
\end{aligned}
$$

If $k=1$ we directly check that

$$
\lambda_{1}^{2} B_{1}-\gamma \lambda_{1}\left(C_{1} \mp A_{1}\right)=0 .
$$

If $k>1$, using (3.20) we obtain

$$
\begin{aligned}
\lambda_{1}^{2} B_{2 k-1}-\gamma \lambda_{1}^{2 k-1}\left(C_{1} \mp A_{1}\right) & =\lambda_{1}^{2}\left( \pm \gamma\left( \pm \lambda_{1}\right)^{2 k-3}\left(C_{1} \mp A_{1}\right)\right)-\gamma \lambda_{1}^{2 k-1}\left(C_{1} \mp A_{1}\right) \\
& =\lambda_{1}^{2} \gamma \lambda_{1}^{2 k-3}\left(C_{1} \mp A_{1}\right)-\gamma \lambda_{1}^{2 k-1}\left(C_{1} \mp A_{1}\right) \\
& =0 .
\end{aligned}
$$

Finally, for any $k \geq 1$ we have

$$
A_{2 k+1}=\lambda_{1}^{2} A_{2 k-1}
$$

Since $A_{1}=-\lambda_{1} \omega\left(e_{1}, e_{3}\right)$ we immediately get (3.17).

In a similar way one may show that

$$
C_{2 k+1}=\lambda_{1}^{2} C_{2 k-1} .
$$

and in consequence (3.18).

To simplify further computations we need to introduce the following notation:

$$
\begin{aligned}
& T_{p, q, r}^{k}(X, Y):=R^{k} \omega(\underbrace{e_{3}, e_{4}, \ldots, e_{3}, e_{4}}_{2 p}, e_{1}, X, \underbrace{e_{3}, e_{4}, \ldots, e_{3}, e_{4}}_{2 q}, e_{1}, Y, \underbrace{e_{3}, e_{4}, \ldots, e_{3}, e_{4}}_{2 r}), \\
& U_{p, q, r}^{k}(X, Y):=-\lambda_{1} h\left(X, e_{3}\right) T_{p, q, r}^{k}\left(e_{4}, Y\right)+\lambda_{1} h\left(X, e_{4}\right) T_{p, q, r}^{k}\left(e_{3}, Y\right), \\
& \hat{U}_{p, q, r}^{k}(X, Y):=-\lambda_{1} h\left(X, e_{3}\right) T_{p, q, r}^{k}\left(Y, e_{4}\right)+\lambda_{1} h\left(X, e_{4}\right) T_{p, q, r}^{k}\left(Y, e_{3}\right) .
\end{aligned}
$$

where $k \geq 1, p, q, r \geq 0, p+q+r=k-1$.

We have the following lemma:

Lemma 3.11. If $S$ and $h$ are of the form (3.3) and $\alpha= \pm \gamma$ and $\beta=\mp \gamma$ then for every $k \geq 1, q, r \geq 0, q+r=k$ and $X, Y \in\left\{e_{3}, e_{4}\right\}$ we have

$$
T_{0, q, r}^{k+1}(X, Y)=\sum_{i=0}^{q-1} U_{i, q-1-i, r}^{k}(X, Y)+\sum_{i=0}^{r-1} \hat{U}_{q, i, r-1-i}^{k}(X, Y) .
$$

Note that it may happen that $q=0$ (respectively $r=0$ ) in such case the sum $\sum_{i=0}^{q-1}$ (respectively the sum $\sum_{i=0}^{r-1}$ ) is not present in the above formula. 
Proof. We compute

$$
\begin{aligned}
T_{0, q, r}^{k+1}(X, Y)= & R\left(e_{1}, X\right) \cdot R^{k} \omega(\underbrace{e_{3}, e_{4}, \ldots, e_{3}, e_{4}}_{2 q}, e_{1}, Y, \underbrace{e_{3}, e_{4}, \ldots, e_{3}, e_{4}}_{2 r}) \\
= & -R^{k} \omega(\underbrace{R\left(e_{1}, X\right) e_{3}, e_{4}, \ldots, e_{3}, e_{4}}_{2 q}, e_{1}, Y, \underbrace{e_{3}, e_{4}, \ldots, e_{3}, e_{4}}_{2 r}) \\
& \ldots \\
& -R^{k} \omega(\underbrace{e_{3}, e_{4}, \ldots, e_{3}, R\left(e_{1}, X\right) e_{4}}_{2 q}, e_{1}, Y, \underbrace{e_{3}, e_{4}, \ldots, e_{3}, e_{4}}_{2 r}) \\
& -R^{k} \omega(\underbrace{e_{3}, e_{4}, \ldots, e_{3}, e_{4}}_{2 q}, R\left(e_{1}, X\right) e_{1}, Y, \underbrace{e_{3}, e_{4}, \ldots, e_{3}, e_{4}}_{2 r}) \\
& -R^{k} \omega(\underbrace{e_{3}, e_{4}, \ldots, e_{3}, e_{4}}_{2 q}, e_{1}, R\left(e_{1}^{e_{1}}, X\right) Y, \underbrace{e_{3}, e_{4}, \ldots, e_{3}, e_{4}}_{2 r}) \\
& -R^{k} \omega(\underbrace{e_{3}, e_{4}, \ldots, e_{3}, e_{4}}_{2 q}, e_{1}, Y, \underbrace{R\left(e_{1}, X\right) e_{3}, e_{4}, \ldots, e_{3}, e_{4}}_{2 r}) \\
& \ldots \\
& -R^{k} \omega(\underbrace{e_{3}, e_{4}, \ldots, e_{3}, e_{4}}_{2 q}, e_{1}, Y, \underbrace{e_{3}, e_{4}, \ldots, e_{3}, R\left(e_{1}, X\right) e_{4}}_{2 r}) .
\end{aligned}
$$

Using the Gauss equation we obtain

$$
\begin{aligned}
T_{0, q, r}^{k+1}(X, Y)= & \sum_{i=0}^{q-1} U_{i, q-1-i, r}^{k}(X, Y) \\
& -R^{k} \omega(\underbrace{e_{3}, e_{4}, \ldots, e_{3}, e_{4}}_{2 q},-S X, Y, \underbrace{e_{3}, e_{4}, \ldots, e_{3}, e_{4}}_{2 r}) \\
& -R^{k} \omega(\underbrace{e_{3}, e_{4}, \ldots, e_{3}, e_{4}}_{2 q}, e_{1}, h(X, Y) \lambda_{1} e_{1}, \underbrace{e_{3}, e_{4}, \ldots, e_{3}, e_{4}}_{2 r}) \\
& +\sum_{i=0}^{r-1} \hat{U}_{q, i, r-1-i}^{k}(X, Y) \\
= & \sum_{i=0}^{q-1} U_{i, q-1-i, r}^{k}(X, Y)+E_{k}^{q+1}(S X, Y)+\sum_{i=0}^{r-1} \hat{U}_{q, i, r-1-i}^{k}(X, Y) .
\end{aligned}
$$

Since $X, Y \in\left\{e_{3}, e_{4}\right\}$ Lemma 3.8 implies (3.22).

Now we can prove

Lemma 3.12. If $S$ and $h$ are of the form (3.3) and $\alpha= \pm \gamma$ and $\beta=\mp \gamma$ then for every $k \geq 1$ we have

$$
T_{p, q, r}^{k}\left(e_{3}, e_{3}\right)=T_{p, q, r}^{k}\left(e_{4}, e_{4}\right)= \pm T_{p, q, r}^{k}\left(e_{3}, e_{4}\right)= \pm T_{p, q, r}^{k}\left(e_{4}, e_{3}\right)
$$

where $p, q, r \geq 0$ and $p+q+r=k-1$

Proof. For $k=1$, by straightforward computations we check that

$$
R \omega\left(e_{1}, e_{3}, e_{1}, e_{3}\right)=R \omega\left(e_{1}, e_{4}, e_{1}, e_{4}\right)= \pm R \omega\left(e_{1}, e_{3}, e_{1}, e_{4}\right)= \pm R \omega\left(e_{1}, e_{4}, e_{1}, e_{3}\right)
$$


SO

$$
T_{0,0,0}^{1}\left(e_{3}, e_{3}\right)=T_{0,0,0}^{1}\left(e_{4}, e_{4}\right)= \pm T_{0,0,0}^{1}\left(e_{3}, e_{4}\right)= \pm T_{0,0,0}^{1}\left(e_{4}, e_{3}\right) .
$$

Assume now that (3.23) is true for some $k \geq 1$ and all $p, q, r \geq 0$ such that $p+q+r=$ $k-1$. We compute

$$
\begin{aligned}
& U_{p, q, r}^{k}\left(e_{3}, e_{3}\right)=-\lambda_{1} T_{p, q, r}^{k}\left(e_{4}, e_{3}\right)=-\lambda_{1} T_{p, q, r}^{k}\left(e_{3}, e_{4}\right) \\
& =U_{p, q, r}^{k}\left(e_{4}, e_{4}\right)= \pm U_{p, q, r}^{k}\left(e_{3}, e_{4}\right)= \pm U_{p, q, r}^{k}\left(e_{4}, e_{3}\right) .
\end{aligned}
$$

In a similar way w get

$$
\hat{U}_{p, q, r}^{k}\left(e_{3}, e_{3}\right)=\hat{U}_{p, q, r}^{k}\left(e_{4}, e_{4}\right)= \pm \hat{U}_{p, q, r}^{k}\left(e_{3}, e_{4}\right)= \pm \hat{U}_{p, q, r}^{k}\left(e_{4}, e_{3}\right) .
$$

Now, let us consider $T_{p, q, r}^{k+1}(X, Y)$ where $p, q, r \geq 0$ and $p+q+r=k$.

If $p=0$ then from Lemma 3.11 we have

$$
T_{0, q, r}^{k+1}(X, Y)=\sum_{i=0}^{q-1} U_{i, q-1-i, r}^{k}(X, Y)+\sum_{i=0}^{r-1} \hat{U}_{q, i, r-1-i}^{k}(X, Y) .
$$

Using (3.24)-(3.25) we obtain

$$
T_{0, q, r}^{k+1}\left(e_{3}, e_{3}\right)=T_{0, q, r}^{k+1}\left(e_{4}, e_{4}\right)= \pm T_{0, q, r}^{k+1}\left(e_{3}, e_{4}\right)= \pm T_{0, q, r}^{k+1}\left(e_{4}, e_{3}\right) .
$$

Assume now that $p>0$. First note that

$$
\begin{aligned}
R^{k} \omega\left(\ldots, R\left(e_{3}, e_{4}\right) e_{3}, e_{4}, \ldots\right) & =R^{k} \omega\left(\ldots,-\gamma e_{3} \pm \gamma e_{4}, e_{4}, \ldots\right) \\
& =-\gamma R^{k} \omega\left(\ldots, e_{3}, e_{4}, \ldots\right) \\
& =R^{k} \omega\left(\ldots, e_{3}, \pm \gamma e_{3}-\gamma e_{4}, \ldots\right) \\
& =-R^{k} \omega\left(\ldots, e_{3}, R\left(e_{3}, e_{4}\right) e_{4}, \ldots\right) .
\end{aligned}
$$

By (3.27) and using the fact that $R\left(e_{3}, e_{4}\right) e_{1}=0$ we get

$$
\begin{aligned}
T_{p, q, r}^{k+1}(X, Y) & R\left(e_{3}, e_{4}\right) \cdot R^{k} \omega(\underbrace{e_{3}, e_{4}, \ldots, e_{3}, e_{4}}_{2 p-2}, e_{1}, X, \underbrace{e_{3}, e_{4}, \ldots, e_{3}, e_{4}}_{2 q}, e_{1}, Y, \underbrace{e_{3}, e_{4}, \ldots, e_{3}, e_{4}}_{2 r}) \\
= & -R^{k} \omega(\underbrace{e_{3}, e_{4}, \ldots, e_{3}, e_{4}}_{2 p-2}, e_{1}, R\left(e_{3}, e_{4}\right) X, \underbrace{e_{3}, e_{4}, \ldots, e_{3}, e_{4}}_{2 q}, e_{1}, Y, \underbrace{e_{3}, e_{4}, \ldots, e_{3}, e_{4}}_{2 r}) \\
& -R^{k} \omega(\underbrace{e_{3}, e_{4}, \ldots, e_{3}, e_{4}}_{2 p-2}, e_{1}, X, \underbrace{e_{3}, e_{4}, \ldots, e_{3}, e_{4}}_{2 q}, e_{1}, R\left(e_{3}, e_{4}\right) Y, \underbrace{e_{3}, e_{4}, \ldots, e_{3}, e_{4}}_{2 r}) \\
= & -T_{p-1, q, r}^{k}\left(R\left(e_{3}, e_{4}\right) X, Y\right)-T_{p-1, q, r}^{k}\left(X, R\left(e_{3}, e_{4}\right) Y\right) .
\end{aligned}
$$

Using (3.12), (3.13) and (3.23), by direct computation, one may check that

$$
T_{p-1, q, r}^{k}\left(R\left(e_{3}, e_{4}\right) X, Y\right)=T_{p-1, q, r}^{k}\left(X, R\left(e_{3}, e_{4}\right) Y\right)=0
$$

for any $X, Y \in\left\{e_{3}, e_{4}\right\}$. In consequence we get

$$
T_{p, q, r}^{k+1}(X, Y)=0
$$

for all $X, Y \in\left\{e_{3}, e_{4}\right\}$. Now from (3.28) and (3.26) we obtain

$$
T_{p, q, r}^{k+1}\left(e_{3}, e_{3}\right)=T_{p, q, r}^{k+1}\left(e_{4}, e_{4}\right)= \pm T_{p, q, r}^{k+1}\left(e_{3}, e_{4}\right)= \pm T_{p, q, r}^{k+1}\left(e_{4}, e_{3}\right)
$$

for every $p, q, r \geq 0, p+q+r=k$. By induction principle the formula (3.23) is true for any $k \geq 1$. 
As an immediate consequence of Lemma 3.12 (see formula (3.28)) we get the following

Corollary 3.13. If $S$ and $h$ are of the form (3.3) and $\alpha= \pm \gamma$ and $\beta=\mp \gamma$ then for every $k \geq 2, p \geq 1, q, r \geq 0$ and $p+q+r=k-1$ we have

$$
T_{p, q, r}^{k}(X, Y)=0
$$

for any $X, Y \in\left\{e_{3}, e_{4}\right\}$.

The above lemmas and corollary alow us to prove the following

Lemma 3.14. If $S$ and $h$ are of the form (3.3) and $\alpha= \pm \gamma$ and $\beta=\mp \gamma$ then for every $k \geq 2$ we have

$$
T_{0,0, k-1}^{k}\left(e_{3}, e_{3}\right)=2^{k-2}\left(\mp \lambda_{1}\right)^{k-1} \gamma \omega\left(e_{3}, e_{4}\right) .
$$

Proof. Let us cosider $T_{0, q, r}^{k+1}\left(e_{3}, e_{3}\right)$, when $q+r=k, k \geq 1$. If $q \geq 1$, using Lemma 3.11 and Corollary 3.13 (if $k \geq 2$ ) we obtain

$$
\begin{aligned}
T_{0, q, r}^{k+1}\left(e_{3}, e_{3}\right) & =\sum_{i=0}^{q-1} U_{i, q-1-i, r}^{k}\left(e_{3}, e_{3}\right)+\sum_{i=0}^{r-1} \hat{U}_{q, i, r-1-i}^{k}\left(e_{3}, e_{3}\right) \\
& =-\lambda_{1} \sum_{i=0}^{q-1} T_{i, q-1-i, r}^{k}\left(e_{4}, e_{3}\right)-\lambda_{1} \sum_{i=0}^{r-1} T_{q, i, r-1-i}^{k}\left(e_{3}, e_{4}\right) \\
& =-\lambda_{1} T_{0, q-1, r}^{k}\left(e_{4}, e_{3}\right) .
\end{aligned}
$$

Now, by Lemma 3.12 we have

$$
T_{0, q, r}^{k+1}\left(e_{3}, e_{3}\right)=-\lambda_{1} T_{0, q-1, r}^{k}\left(e_{4}, e_{3}\right)=\mp \lambda_{1} T_{0, q-1, r}^{k}\left(e_{3}, e_{3}\right)
$$

that is

$$
T_{0, q, r}^{k+1}\left(e_{3}, e_{3}\right)=\left(\mp \lambda_{1}\right)^{q} T_{0,0, r}^{r+1}\left(e_{3}, e_{3}\right) .
$$

If $q=0$, by Lemma 3.11 and Lemma 3.12 we have

$$
T_{0,0, k}^{k+1}\left(e_{3}, e_{3}\right)=-\lambda_{1} \sum_{i=0}^{k-1} T_{0, i, k-1-i}^{k}\left(e_{3}, e_{4}\right)=\mp \lambda_{1} \sum_{i=0}^{k-1} T_{0, i, k-1-i}^{k}\left(e_{3}, e_{3}\right)
$$

for $k \geq 1$. Applying (3.31) to (3.32) we get

$$
T_{0,0, k}^{k+1}\left(e_{3}, e_{3}\right)=\mp \lambda_{1} \sum_{i=0}^{k-1}\left(\mp \lambda_{1}\right)^{i} T_{0,0, k-i-1}^{k-i}\left(e_{3}, e_{3}\right) .
$$

By straightforward computations we check that

$$
T_{0,0,0}^{1}\left(e_{3}, e_{3}\right)=\gamma \omega\left(e_{3}, e_{4}\right) \quad \text { and } \quad T_{0,0,1}^{2}\left(e_{3}, e_{3}\right)=\mp \lambda_{1} \gamma \omega\left(e_{3}, e_{4}\right)
$$


so in particular (3.30) is true for $k=2$. Let us fix $k_{0} \geq 2$ and assume that (3.30) is true for any $k \in\left\{2, \ldots, k_{0}\right\}$. Now we have

$$
\begin{aligned}
T_{0,0, k_{0}}^{k_{0}+1}\left(e_{3}, e_{3}\right)= & \mp \lambda_{1} \sum_{i=0}^{k_{0}-2}\left(\mp \lambda_{1}\right)^{i} 2^{k_{0}-i-2}\left(\mp \lambda_{1}\right)^{k_{0}-i-1} \gamma \omega\left(e_{3}, e_{4}\right) \\
& +\left(\mp \lambda_{1}\right)^{k_{0}} T_{0,0,0}^{1}\left(e_{3}, e_{3}\right) \\
= & \left(\mp \lambda_{1}\right)^{k_{0}} \gamma \omega\left(e_{3}, e_{4}\right) \sum_{i=0}^{k_{0}-2} 2^{k_{0}-i-2}+\left(\mp \lambda_{1}\right)^{k_{0}} \gamma \omega\left(e_{3}, e_{4}\right) \\
= & 2^{k_{0}-1}\left(\mp \lambda_{1}\right)^{k_{0}} \gamma \omega\left(e_{3}, e_{4}\right) .
\end{aligned}
$$

That is (3.30) holds also for $k=k_{0}+1$. Now, by induction principle (3.30) is true for any $k \geq 2$.

Now we are ready to prove main results of this paper. Namely we have

Theorem 3.15. Let $f: M \rightarrow \mathbb{R}^{5}$ be a non-degenerate affine hypersurface with a locally equiaffine transversal vector field $\xi$ and an almost symplectic form $\omega$. If $R^{k} \omega=0$ for some $k \geq 1$ and the second fundamental form is Lorentzian on $M$ (that is has signature $(3,1)$ ) then the shape operator $S$ has the rank $\leq 1$.

Proof. Let $x \in M$ and let $\left\{e_{1}, \ldots, e_{4}\right\}$ be the basis from Lemma 3.2, If $S$ and $h$ are of the form (3.2), then in the same way as in the proof of Theorem 1.2 (see [19] for details) we obtain that $S$ is equal to zero thus rank $S_{x}=0$.

Let $S$ and $h$ have the form (3.3). If $\beta^{2}-\gamma^{2} \neq 0$ then by Lemma 3.5 we can change the basis $\left\{e_{1}, \ldots, e_{4}\right\}$ of $T_{x} M$ to $h$-ortonormal basis $e_{1}^{\prime}, \ldots, e_{4}^{\prime}$ in such a way that $S$ and $h$ are of the form (3.7). In particular, $S$ is diagonal. Since $\beta^{2}-\gamma^{2} \neq 0$, Corollary 3.4 implies that $\alpha+\beta \neq 0$, thus $\operatorname{rank} S_{x} \geq 1$. However, since $S$ is diagonal we again can use methods from [19] (proof of Theorem 1.2) and show that $\operatorname{rank} S_{x}=0$, what leads to contradiction. It means that the case $\beta^{2}-\gamma^{2} \neq 0$ is not possible.

Assume now that $\beta^{2}-\gamma^{2}=0$. By Corollary 3.4 we have $\alpha \beta+\gamma^{2}=0$ and in consequence we get that $\alpha= \pm \gamma$ and $\beta=\mp \gamma$. Without loss of generality (rearranging $e_{1}$ and $e_{2}$ if needed) we may always assume that $\left|\lambda_{1}\right| \geq\left|\lambda_{2}\right| \geq 0$. If $\lambda_{1}=\lambda_{2}=0$ then $\operatorname{rank} S_{x}=1$ (since $\gamma \neq 0$ ) and the proof is completed. Let as assume that $\lambda_{1} \neq 0$. Since $R^{k} \omega=0$ for some $k \geq 1$ then in particular $R^{2 k} \omega=0$ and $R^{2 k+1} \omega=0$. Now by Lemma 3.10 we immediately obtain $\omega\left(e_{1}, e_{3}\right)=\omega\left(e_{1}, e_{4}\right)=0$. Since $\omega$ is non-degenerate then

$$
\begin{aligned}
\operatorname{det} \omega & =\left(\omega\left(e_{1}, e_{2}\right) \omega\left(e_{3}, e_{4}\right)-\omega\left(e_{1}, e_{3}\right) \omega\left(e_{2}, e_{4}\right)+\omega\left(e_{1}, e_{4}\right) \omega\left(e_{2}, e_{3}\right)\right)^{2} \\
& =\left(\omega\left(e_{1}, e_{2}\right) \omega\left(e_{3}, e_{4}\right)\right)^{2} \neq 0 .
\end{aligned}
$$

In particular $\omega\left(e_{3}, e_{4}\right) \neq 0$. Now Lemma 3.14 implies that $\lambda_{1} \gamma=0$ what (since $\gamma \neq 0$ and $\lambda_{1} \neq 0$ ) leads us to contradiction. Summarising we must have $\lambda_{1}=0$ and in consequence also $\lambda_{2}=0$.

From Theorem 3.15 we directly obtain the following

Theorem 3.16. Let $f: M \rightarrow \mathbb{R}^{5}$ be a non-degenerate affine hypersurface with a locally equiaffine transversal vector field $\xi$ and an almost symplectic form $\omega$. If $\nabla^{k} \omega=0$ for some $k \geq 1$ and the second fundamental form is Lorentzian on $M$ (that is has signature $(3,1)$ ) then the shape operator $S$ has the rank $\leq 1$. 
Proof. If $\nabla^{k} \omega=0$ for some $k$ then, of course, we have that also $\nabla^{2 k} \omega=0$ and now by Lemma 3.1 we get $R^{k} \omega=0$. Now, thesis is an immediate consequence of Theorem 3.15 .

This Research was financed by the Ministry of Science and Higher Education of the Republic of Poland.

\section{REFERENCES}

[1] Albuquerque, R., Picken, R.: On invariants of almost symplectic connections, Math. Phys. Anal. Geom. 18 (2015).

[2] Bokan, N., Nomizu, K., Simon, U.: Affine hypersurfaces with parallel cubic forms, Tohoku Math. J. 42 (1990), 101108.

[3] O. Baues and V. Cortés, Realisation of special Kähler manifolds as parabolic spheres, Proc. Amer. Math. Soc. 129 (2001), 2403-2407.

[4] Belkhelfa, M., Dillen, F., Inoguchi J.: Surfaces with parallel second fundamental form in Bianchi-Cartan-Vranceanu spaces,PDEs, Submanifolds and Affine Differential Geometry, Banach Center Publications 57 (2002), 67-87.

[5] V. Cortés, M.-A. Lawn and L. Schäfer, Affine hyperspheres associated to special para-Kähler manifolds, International Journal of Geometric Methods in Modern Physics 3 (2006), 9951010.

[6] Cortés, V., Mayer, C., Mohaupt,T., Saueressig, F.: Special geometry of Euclidean supersymmetry I: Vector multiplets, J. High Energy Phys. 403 (2004), 028-0312001.

[7] Dillen, F.: The classification of hypersurfaces of a Euclidean space with parallel higher order fundamental form, Math. Z. 203 (1990), 635-643.

[8] Dillen, F.: Hypersurfaces of a real space form with parallel higher order fundamental form, Soochow J. Math. 18 (1992), 321-338.

[9] Dillen, F., Vrancken, L.: 3-Dimensional affine hypersurfaces in $R^{4}$ with parallel cubic form, Nagoya Math. J. 124 (1991), 4153.

[10] Dillen, F., Vrancken, L., Yaprak, S.: Affine hypersurfaces with parallel cubic form, Nagoya Math. J. 135 (1994), 153164.

[11] Freed, D. S.: Special Kähler manifolds, Commun. Math. Phys. 203 (1999), 31-52.

[12] Hildebrand, R.: Centro-affine hypersurface immersions with parallel cubic form, Beiträge zur Algebra und Geometrie / Contributions to Algebra and Geometry 56 (2015), 593-640.

[13] Hu, Z.J., Li, C.C., Li, H., Vrancken, L.: Lorentzian affine hypersurfaces with parallel cubic form, Results Math. 59 (2011), 577620.

[14] Hu, Z.J., Li, C.C., Li, H., Vrancken, L.: The classification of 4-dimensional non-degenerate affine hypersurfaces with parallel cubic form, J. Geom. Phys. 61 (2011), 2035-2057.

[15] Hu, Z.J., Li, H., Vrancken, L.: Locally strongly convex affine hypersurfaces with parallel cubic form, J. Differ. Geom. 87 (2011), 239307.

[16] Kon, M.: Hypersurfaces with Almost Complex Structures in the Real Affine Space, Colloquium Mathematicum 108 (2007) 329-338

[17] Lumiste, U.: Submanifolds with parallel fundamental form, Handbook of Differential Geometry Vol. 1, Elsevier Science B.V., Amsterdam (2000), 779-864.

[18] Nomizu, K., Sasaki, T.: Affine differential geometry, Cambridge University Press (1994)

[19] Szancer, M.: On Affine Hypersurfaces with an Almost Symplectic Structure, Results Math 71 (2017) 377-396

[20] Szancer, M.: Lorentzian affine hypersurfaces with an almost symplectic form. Journal of Geometry and Physics 119 (2017) 171-186

[21] Szancer, M.: On some generalization of special affine hyperspheres, Colloq. Math. 149 (2017), 239-256.

[22] Szancer, Z.: Real Hypersurfaces with Parallel Induced Almost Contact Structures, Ann. Polon. Math. 104(2) (2012), 203-215.

[23] Szancer, Z.: Parallel Almost Paracontact Structures on Affine Hypersurfaces, arXiv:1805.08054 (2018).

[24] Van der Veken, J.: Higher Order Parallel Surfaces in Bianchi-Cartan-Vranceanu Spaces, Results Math. 51 (2008), 339-359. 
[25] Vrancken, L.: Affine higher order parallel hypersurfaces, Ann. Fac. Sci. Toulouse Math. 9 (1988) 341-353

[26] Vrancken, L.: Affine surfaces with higher order parallel cubic form, Tohoku Math. J. 43 (1991), 127-139.

Michal Szancer, Gornikow Street 21/1, 30-819 Krakow, Poland

E-mail address: mszancer@gmail.com

Zuzanna Szancer, Department of Applied Mathematics, University of Agriculture in Krakow, 253c Balicka Street, 30-198 Krakow, Poland

E-mail address: Zuzanna.Szancer@urk.edu.pl 